\title{
The effects of vitamin D3 on lipogenesis in the liver and adipose tissue of pregnant rats
}

\author{
EUN-JIN KANG ${ }^{1}$, JAE-EON LEE ${ }^{1}$, SUNG-MIN AN $^{1}$, JAE HO LEE ${ }^{1}$, HYEOG SOONG KWON ${ }^{2}$, \\ BYOUNG CHUL KIM ${ }^{2}$, SEON JONG KIM ${ }^{2}$, JOO MAN KIM ${ }^{2}$, DAE YOUN HWANG ${ }^{1}$, YOUNG-JIN JUNG ${ }^{1}$, \\ SEUNG YUN YANG ${ }^{1}$, SEUNG CHUL KIM ${ }^{3}$ and BEUM-SOO AN ${ }^{1}$ \\ ${ }^{1}$ Department of Biomaterials Science and ${ }^{2}$ Applied IT and Engineering, College of Natural Resources and \\ Life Science/Life and Industry Convergence Research Institute, Pusan National University, \\ Gyeongsangnam-do; ${ }^{3}$ Department of Obstetrics and Gynecology, Biomedical Research Institute, \\ Pusan National University School of Medicine, Busan, Republic of Korea
}

Received March 10, 2015; Accepted July 15, 2015

DOI: $10.3892 / \mathrm{ijmm} .2015 .2300$

\begin{abstract}
Obesity is a worldwide individual and public health issue, and contributes to the development of numerous chronic diseases. In particular, maternal obesity has harmful effects on both the mother and child during and after pregnancy. The digestion and metabolism of food are controlled by endocrine factors, including insulin, glucagon and estrogen. These hormonal factors are differentially regulated during pregnancy due to the specialized hormonal environment during this period. In the present study, we examined the effects of 1,25-dihydroxyvitamin D3 (VD3), an active hormonal form of nutritional vitamin D3, on lipid metabolism in pregnant rats. The body weight of rats treated with VD3 was significantly reduced compared to that of the rats in the control group. In addition, histological analysis demonstrated that the amount of fat stored in adipocytes was reduced by treatment with VD3. To determine the role of VD3 in lipid metabolism, the expression levels of lipid metabolism-associated genes were measured in the rat adipose tissue and liver. VD3 negatively regulated the expression of various lipogenic genes, including fatty acid synthase (FAS), stearoyl-CoA desaturase 1 (SCD1) and acetyl-CoA carboxylase 1 (ACC1), in both the adipose tissue and liver. However, the regulators of lipogenic enzymes such as, sterol regulatory element-binding protein-1c (SREBP-1c), peroxisome proliferator-activated receptor- $\gamma$ (PPAR- $\gamma$ ) and insulin-induced gene 2 (INSIG2) were differentially regulated by VD3 in a tissue-specific manner. On the whole,
\end{abstract}

Correspondence to: Professor Beum-Soo An, Department of Biomaterials Science, College of Natural Resources and Life Science, Pusan National University, 50 Cheonghak-ri, Samrangjin-eup, Miryang, Gyeongsangnam-do 627-706, Republic of Korea

E-mail: anbs@pusan.ac.kr

Key words: vitamin D, lipogenesis, liver, adipose tissue, pregnancy these findings suggest that VD3 regulates lipid metabolism and deposition in the liver and adipose tissue, and thereby reduces fat in pregnant animals, as well as body weight. Our results suggest that the alteration of lipogenesis through the administration of VD3 may help to reduce excessive weight gain during pregnancy and prevent obesity-related pregnancy complications such as pre-eclampsia, gestational diabetes, hypertension and issues with labor.

\section{Introduction}

Vitamin D, which comprises a group of fat-soluble secosterols found naturally in very few foods, is photosynthesized in the skin of vertebrates by the action of solar ultraviolet-B (UV-B) radiation (1). The major biological function of vitamin $\mathrm{D}$ is to maintain normal serum concentrations of calcium and phosphorus by enhancing the ability of the small intestine to absorb these minerals from food (1). The original active form of vitamin $\mathrm{D}$ is derived from cholesterol. Hepatic hydroxylation enzymes and possibly other enzymes, convert cholesterol into the major circulating metabolite 25-hydroxyvitamin D (25D), which is a relatively long-lived metabolite, as well as a marker of the vitamin D status. $25 \mathrm{D}$ is hydroxylated at $1 \alpha$ in the kidneys and peripheral tissue to produce the hormonally active form of vitamin D, 1,25-dihydroxyvitamin D3 (VD3) (2-4). The majority of the pleiotropic, long-term effects of VD3 are mediated by its binding to a nuclear high-affinity receptor [vitamin D receptor (VDR)], which is principally located in the nuclei of target cells, in complex with 9-cis-retinoic acid, a retinoid $\mathrm{X}$ receptor (RXR) (5). The activated VDR-RXR complex binds to a vitamin $\mathrm{D}$ response element (VDRE) located in the promoters of target genes that are involved in calcium absorption, such as transient receptor potential cation channel, subfamily V (TRPV)5 and TRPV6 genes (6).

It has now become clear that vitamin $\mathrm{D}$ has additional physiological functions beyond calcium absorption. Previous research has provided evidence indicating that vitamin D signaling regulates several physiological responses unrelated to mineral 
ion homeostasis, including both innate and adaptive immune responses (7). Moreover, epidemiological data have established associations between a lack of UV-B exposure, vitamin D defficiency and the prevalence of certain types of cancer (8-10). Vitamin D is also related to lipid metabolism and obesity. Indeed, epidemiologically, obesity has been found to be associated with lower serum levels of 25D and sun exposure $(11,12)$.

According to previous data, VD3 is known to inhibit mouse 3T3-L1 pre-adipocyte differentiation, which is mediated by the induction of the expression of a number of genes, such as CCAAT/enhancer-binding protein- $\alpha$ (C/EBP- $\alpha)$, peroxisome proliferator-activated receptor- $\gamma(\operatorname{PPAR}-\gamma)$ and sterol regulatory element-binding protein-1c (SREBP-1c), which are characteristic of mature adipocytes. However, the effects of VD3 on mouse pre-adipocytes are limited in blocking fat cell differentiation and gene expression within the first $48 \mathrm{~h}$ of initiating differentiation (13).

Lipogenesis and adipose tissue development are regulated by female sex steroid hormones. The adipocytes of female mice have increased lipogenic capacities compared with those of male mice, although adipocytes the of females are smaller than those of males (14). In addition, the female sex hormone, estrogen, regulates glucose homeostasis, which is a crucial factor in fatty acid metabolism $(14,15)$.

During gestation, women have a special endocrine environment which helps to maintain pregnancy, and thereby lipid metabolism is regulated differently from that of a non-pregnant women. Since more nutrients are required, the endocrine system tends to ncrease fat accumulation in the pregnant woman (16). The proliferation of adipose tissue is often accompanied by a state of insulin resistance during pregnancy. This pregnancy-specific adaptive response allows glucose to be transferred across the placenta more efficiently and thus fetal growth is promoted (17). However, this response may also result in the mother and fetus developing elevated blood glucose and insulin levels, which in turn means a greater risk of obesity. Certain studies have indicated that birth weight is directly associated with the BMI of the baby, which implies that gestational diabetes in the mother contributes to obesity in her child (18-20). Furthermore, maternal obesity increases the risk of other pregnancy complications, including preeclampsia, gestational diabetes, hypertension, thrombosis and labor issues (21-23).

Certain studies have suggested that the serum concentration of vitamin $\mathrm{D}$ differs between pregnant and non-pregnant women, indicating that vitamin D may play a specific role during pregnancy $(24,25)$. However, correlations between lipogenesis and vitamin D during pregnancy have not yet been established. Therefore, in the present study, we examined the effects of VD3 on lipogenesis and fat accumulation in the liver and adipose tissue of pregnant rats.

\section{Materials and methods}

Reagents and chemicals. VD3 was purchased from Merck Millipore Co. (Billerica, MA, USA). Corn oil and mouse anti- $\beta$ actin antibody were purchased from Sigma-Aldrich (St. Louis, MO, USA). Rabbit anti-FAS (sc-20140), mouse anti-SREBP-1c (sc-133551) and mouse anti-PPAR- $\gamma$ (sc-7273) antibodies, and horseradish peroxidase (HRP)-conjugated anti-rabbit (sc-2313) and anti-mouse (sc-2005) IgG were all purchased from Santa Cruz Biotechnology, Inc. (Santa Cruz, CA, USA).

Animals and treatments. Pregnant Sprague-Dawley (SD; n=12) female rats were purchased from Samtako (Osan, Korea) and handled at the Pusan National University Laboratory Animal Resources Center (Busan, Korea), which is accredited by the Korea FDA according to the National Institutes of Health guidelines. The rats were housed in cages under a 12-h light/dark cycle, with a constant temperature of $23 \pm 1^{\circ} \mathrm{C}$. The rats were fed a diet of soy-free pellets (Samtako). All experimental animal procedures were approved by the Ethics Committee of Pusan National University (Busan, Korea; approval no. PNU-2013-0401). From gestational days (GD) 11-16, the rats were administered daily subcutaneous injections of VD3 $(1 \mu \mathrm{g} / \mathrm{kg} /$ day $)$ or corn oil as the vehicle control. The dosage was adjusted according to changes in body weight (BW). All the pregnant female rats were sacrificed on GD 17 using $\mathrm{CO}_{2}$ gas. Tissue samples (liver and abdominal adipose) and fetus were removed using scissors from each individual pregnant rat and stored at $-70^{\circ} \mathrm{C}$ or fixed in $10 \%$ formalin until analysis.

Reverse transcription-quantitative PCR (RT-qPCR). Total RNA was extracted using TRIzol reagent (Invitrogen, Carlsbad, CA, USA) according to the manufacturer's instructions. The concentration of total RNA was measured using a spectrophotometer (Biospec-nano; Shimadzu, Kyoto, Japan). First-strand complementary DNA (cDNA) was prepared from total RNA $(3 \mu \mathrm{g})$ by reverse transcription using Moloney murine leukemia virus (M-MLV) reverse transcriptase (Invitrogen) and random primers (9-mers; Takara Bio Inc., Shiga, Japan). Quantitative PCR (qPCR) was performed using a cDNA template $(2 \mu \mathrm{l})$ and $2 \mathrm{X}$ Power SYBR-Green (6 $\mu \mathrm{l}$; Toyobo Co., Ltd., Osaka, Japan) containing specific primers. The primer sequences for fatty acid synthase (FAS), stearoyl-CoA desaturase 1 (SCD1), acetyl-CoA carboxylase 1 (ACC1), SREBP-1c, PPAR- $\gamma, \mathrm{C} / \mathrm{EBP}-\alpha$ and insulin-induced gene 2 (INSIG2) are listed in Table I. qPCR was carried out for 40 cycles using the following parameters: denaturation at $95^{\circ} \mathrm{C}$ for $15 \mathrm{sec}$, followed by annealing and extension at $70^{\circ} \mathrm{C}$ for $60 \mathrm{sec}$. The fluorescence intensity was measured at the end of the extension phase of each cycle. The threshold value for the fluorescence intensity of all samples was set manually. The reaction cycle at which the PCR products exceeded this fluorescence intensity threshold during the exponential phase of PCR amplification was considered to be the threshold cycle (CT). The expression of the target gene was quantified relative to that of $\beta$-actin, an ubiquitous housekeeping gene, based on a comparison of CTs at a constant fluorescence intensity.

Western blot analysis. Protein samples were extracted from the liver and adipose tissue using PRO-PREP solution (Intron Biotechnology, Seoul, Korea) following the manufacturer's instructions. A total of $40 \mu \mathrm{g}$ cytosolic proteins was separated by $8-10 \%$ sodium dodecyl sulfate polyacrylamide gel electrophoresis (SDS-PAGE) and transferred onto nitrocellulose membranes (Daeil Lab Service Co., Ltd., Seoul, Korea). The membranes were then blocked for $1 \mathrm{~h}$ with $5 \%$ skim milk (Difco, Sparks, MD, USA) in phosphate-buffered saline (PBS) with $0.05 \%$ Tween-20 (PBS-T). After blocking, the membranes were 
Table I. Primer sequences used for RT-qPCR.

\begin{tabular}{llc}
\hline $\begin{array}{l}\text { Gene } \\
\text { name }\end{array}$ & \multicolumn{1}{c}{\begin{tabular}{c}
\multicolumn{1}{c}{$\begin{array}{c}\text { Primer sequence } \\
\left(5^{\prime} \rightarrow 3^{\prime}\right)\end{array}$} \\
FAS
\end{tabular}} & $\begin{array}{c}\text { Fragment } \\
(\mathrm{bp})\end{array}$ \\
& $\begin{array}{l}\text { F: CCAGGGTTTGGAATTATTC } \\
\text { RCD1 GAGATAAACCCTAAGGCTC }\end{array}$ & 293 \\
& F: AAGAGCATTTTTCAACCCTA & 314 \\
ACC1 & R: GTCTCAGAATTTGCTTTATT & \\
& F: CTTGGCCTACTGGCTCTGAC & 216 \\
SREBP-1c & F: TTCTTCGTGCAGATGTGGAG & 234 \\
& R: TAACGAGCAGAGCAGCAGAA & \\
PPAR- $\gamma$ & F: TGAAAAGCGGTGTGAGACTG & 220 \\
& R: TGATGGGTCCAAAATTCCAT & \\
C/EBP- $\alpha$ & F: ACCTGTGAGACGACGCTCTT & 160 \\
& R: GCCGGTGACTCAGAGATAGC & \\
INSIG2 & F: CCTTGGTGTTGGAATTGCTT & 167 \\
& R: TTGCTAGCTGACGACCAATG &
\end{tabular}

F, forward; R, reverse; SREBP-1c, sterol regulatory element-binding protein-1c; INSIG2, insulin induced gene 2; PPAR- $\gamma$, peroxisome proliferator-activated receptor- $\gamma$; C/EBP- $\alpha$, CCAAT/enhancer-binding protein- $\alpha$; FAS, fatty acid synthase; SCD1, stearoyl-CoA desaturase 1; ACC1, acetyl-CoA carboxylase 1.

incubated $\mathrm{o} / \mathrm{n}$ with antibodies specific for FAS (dilution 1:500), SREBP-1c (dilution 1:500) and PPAR- $\gamma$ (dilution 1:500), as well as with HRP-conjugated anti-rabbit and anti-mouse secondary antibodies (dilution 1:2,000) in 5\% skim milk with PBS-T for 1 h. Luminol reagent (Bio-Rad Laboratories Inc., Hercules, CA, USA) was used to visualize antibody binding. Each blot was then stripped by incubation with $2 \%$ SDS and $100 \mathrm{mM} 2$-mercaptoethanol in $62.5 \mathrm{mM}$ Tris- $\mathrm{HCl}$ (pH 6.8) for $30 \mathrm{~min}$ at $50-60^{\circ} \mathrm{C}$. The membranes were subsequently probed with an antibody against $\beta$-actin (diluted 1:2,000; Santa Cruz Biotechnology, Inc.) as an internal control. The blots were scanned using Gel Doc 1000, version 1.5 (Bio-Rad Laboratories Inc.) and the band intensities were normalized to $\beta$-actin levels.

Histological analysis. Adipose tissue was removed from the rats using scissors, immediately fixed with $10 \%$ formalin, embedded in paraffin wax, routinely processed, and then sectioned into 4- $\mu \mathrm{m}$-thick sections. The tissue sections were then stained with hematoxylin and eosin (H\&E; SigmaAldrich). Five different areas from each animal were analyzed to determine the histological changes. Images of the tissues were captured at $\mathrm{x} 20$ magnification using an optical microscope (model BX50F-3; Olympus, Tokyo, Japan).

Statistical analyses. The results are presented as the means \pm standard deviation (SD). Data were analyzed using one-way analysis of variance (ANOVA) (SPSS for Windows, 10.10, standard version; SPSS Inc., Chicago, IL, USA). P-values <0.05 were considered to indicate statistically significant differences.

\section{Results}

Changes in body weight in the pregnant rats. To determine the effects of VD3 on fat accumulation during pregnancy, the rats were subcutaneously injected with $1 \mu \mathrm{g} / \mathrm{kg}$ /day of VD3, from GD11 to GD16, and were then sacrificed on GD17. The body weights of the rats were measured on GD17 and normalized to the values obtained on GD11; the changes in body weight are shown in Fig. 1A. Corn oil was used as a negative control. We observed that VD3 significantly decreased body weight by up to $25 \%$ compared with the controls. To examine the possible adverse effects of VD3 on the fetus, fetal body weight was also measured. The body weight of the fetuses was not altered significantly (Fig. 1B).

Histological changes in adipose tissues following treatment with VD3. As VD3 was shown to reduce body weight, we then analyzed the histological changes in adipose tissue following treatment with VD3 in order to determine whether the decrease in body weight was due to the loss of fatty tissue. The adipose tissue was fixed in paraffin and stained with H\&E. Images of the stained adipocytes were then captured using an optical microscope, and the number of cells in the diagram was counted. The fat deposition rate was calculated based on the number of adipocytes. Five separate areas from each animal were analyzed, and the results are shown in Fig. 1C. The fat deposition rate in adipose tissue was decreased following treatment with VD3 compared with the vehicle-treated controls (Fig. 1C). We also noted that the size of the adipocytes was smaller in the VD-treated tissues than those treated with the vehicle control. These results suggest that VD3 treatment reduces fat deposition, resulting in a decrease in total body weight of the pregnant rats.

Regulation of lipogenesis-associated gene expression in the liver. To determine the mechanisms through which VD3 regulates fat deposition and body weight, the expression levels of genes associated with lipogenesis, so-called lipogenic enzymes, were measured in the liver. The liver is an organ that biosynthesizes and catalyzes triacylglycerol (TG), which is the typical form of animal fat (26). Therefore, the amount of lipogenic enzymes in the liver is important for fat synthesis. Constitutive lipogenic enzymes in the liver include FAS, SCD1 and ACC1 (27). We found that VD3 significantly reduced the mRNA expression of FAS in the liver by up to $80 \%$ (Fig. 2). The levels of the other lipogenic enzymes, SCD1 and ACC1, were also markedly decreased following treatment with VD3. It is known that lipogenic enzymes are controlled at the transcriptional level and that proadipogenic transcription factors, such as SREBP-1c, PPAR- $\gamma$ and C/EBP- $\alpha$ are involved in the regulation of adipocyte formation (28). Therefore, in this study, we measured the mRNA expression of SREBP-1c, PPAR- $\gamma$ and C/EBP- $\alpha$ in the liver (Fig. 2). The mRNA expression levels of SREBP-1c were significantly decreased following treatment with VD3, and a similar result was noted in relation to the expression levels of its target genes, FAS, SCD1 and ACC1. However, the mRNA levels of PPAR $-\gamma$ and C/EBP- $\alpha$ were slightly upregulated following treatment with VD3. The transcripts of PPAR- $\gamma$ and C/EBP were slightly elevated following treatment with VD3 without 

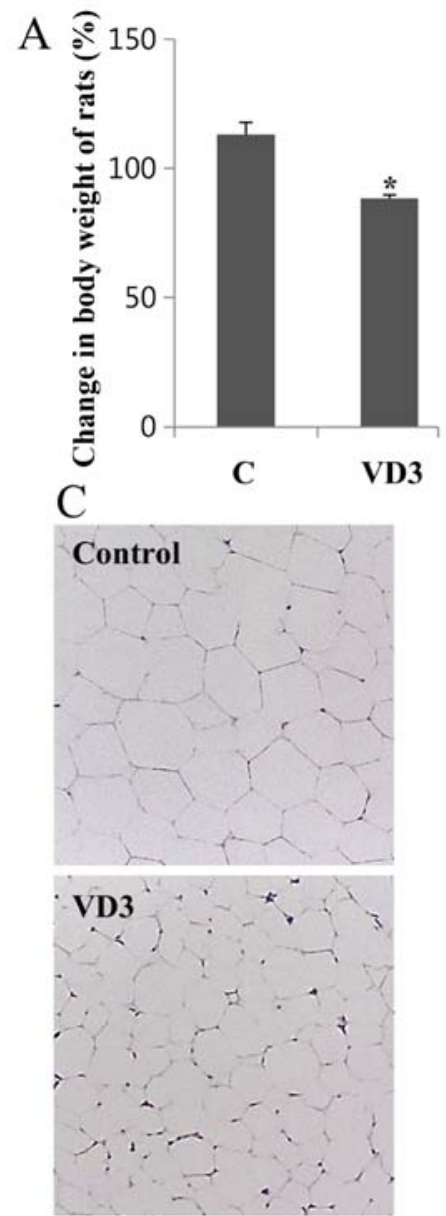

B
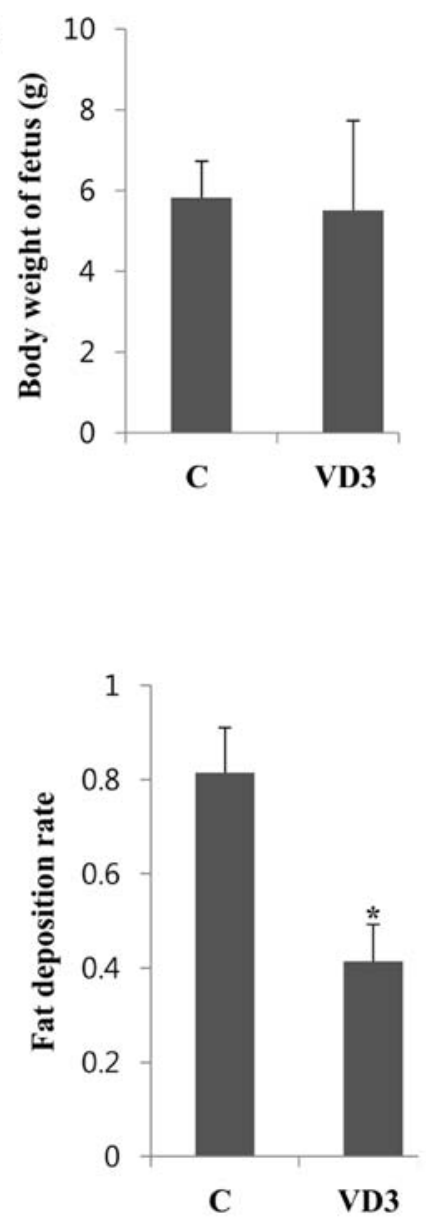

Figure 1. Effect of 1,25-dihydroxyvitamin D3 (VD3) on body weight (BW) of pregnant rats and fat deposition. Pregnant rats were treated with VD3 (1 $\mu \mathrm{g} / \mathrm{kg} / \mathrm{day}$ ). (A) Changes in BW of pregnant rats and (B) changes in the body weight of the fetus were determined. Adipose tissue was stained with $\mathrm{H} \& \mathrm{E}$, and the fat deposition rate was analyzed based on the number of adipocytes (C). Data are expressed as the means \pm SD of 5-6 animals. " $\mathrm{P}<0.05$ compared to the control (C; vehicle-treated) group.

significance. These results suggest that the expression levels of lipogenic enzymes in the liver is reduced by VD3 treatment in pregnant rats, and this is possibly mediated by SREBP-1c. To confirm the regulation of these genes at the translational level, we examined the protein expression levels of FAS, SREBP-1c and PPAR- $\gamma$ by western blot analysis in the absence (vehicle control) and presence of VD3 (Fig. 3). We selected 3 independent protein samples from the control and VD3 groups for western blot analysis, and the results are represented in (Fig. 3). The protein expression of FAS and SREBP-1c in the liver was significantly decreased following treatment with VD3 compared with the control. These results were in agreement with the mRNA results (Fig. 2), indicating that VD3 reduced the expression of FAS and SREBP-1c at both the transcriptional and translational level. The basal expression of PPAR- $\gamma$ was relatively low and was not altered significantly following treatment with VD3 (Fig. 3).

Regulation of lipogenesis-associated gene epxression in adipose tissue. We then examined the effects of VD3 on the expression of lipogenic enzymes, as well as their transcriptional regulators in adipose tissue, where fat is mainly stored (Fig. 4). Treatment with VD3 markedly downregulated the lipogenic enzymes, FAS, SCD1 and ACC1, in adipose tissue, decreasing their expresion by up to $90 \%$ (Fig. 4). These results are similar to those involving the liver (Fig. 2), suggesting that VD3 modulates the expression of lipogenic enzymes in both the liver and adipose tissue. The transcriptional levels of PPAR- $\gamma$ were significantly upregulated following treatment with VD3, which was slightly different to the results obtained with the liver (the mRNA levels of PPAR- $\gamma$ were only slightly increased in the liver). The regulation of SREBP-1c expression in adipose tissue also differed from that in the liver. The mRNA expression of SREBP-1c in adipose tissue was not significantly altered following treatment with VD3, whereas it was greatly reduced in the liver. The protein levels of FAS, SREBP-1c and PPAR- $\gamma$ were regulated in a similar manner to the mRNA levels (Fig. 5). Treatment with VD3 markedly downregulated the protein expression of FAS, whereas, it did not significantly alter that of SREBP-1c. PPAR- $\gamma$ protein expression increased following treatment with VD3, which was similar to the results of mRNA expression.

Tissue-specific regulation of INSIG2 by VD3 in the liver and adipose tissue of pregnant rats. Since PPAR- $\gamma$ and SREBP-1c were differentially regulated by VD3, the expression of INSIG2, an intermediate regulator between PPAR- $\gamma$ and SREBP-1c, was investigated in the liver and adipose tissue (Fig. 6). VD3 modu- 

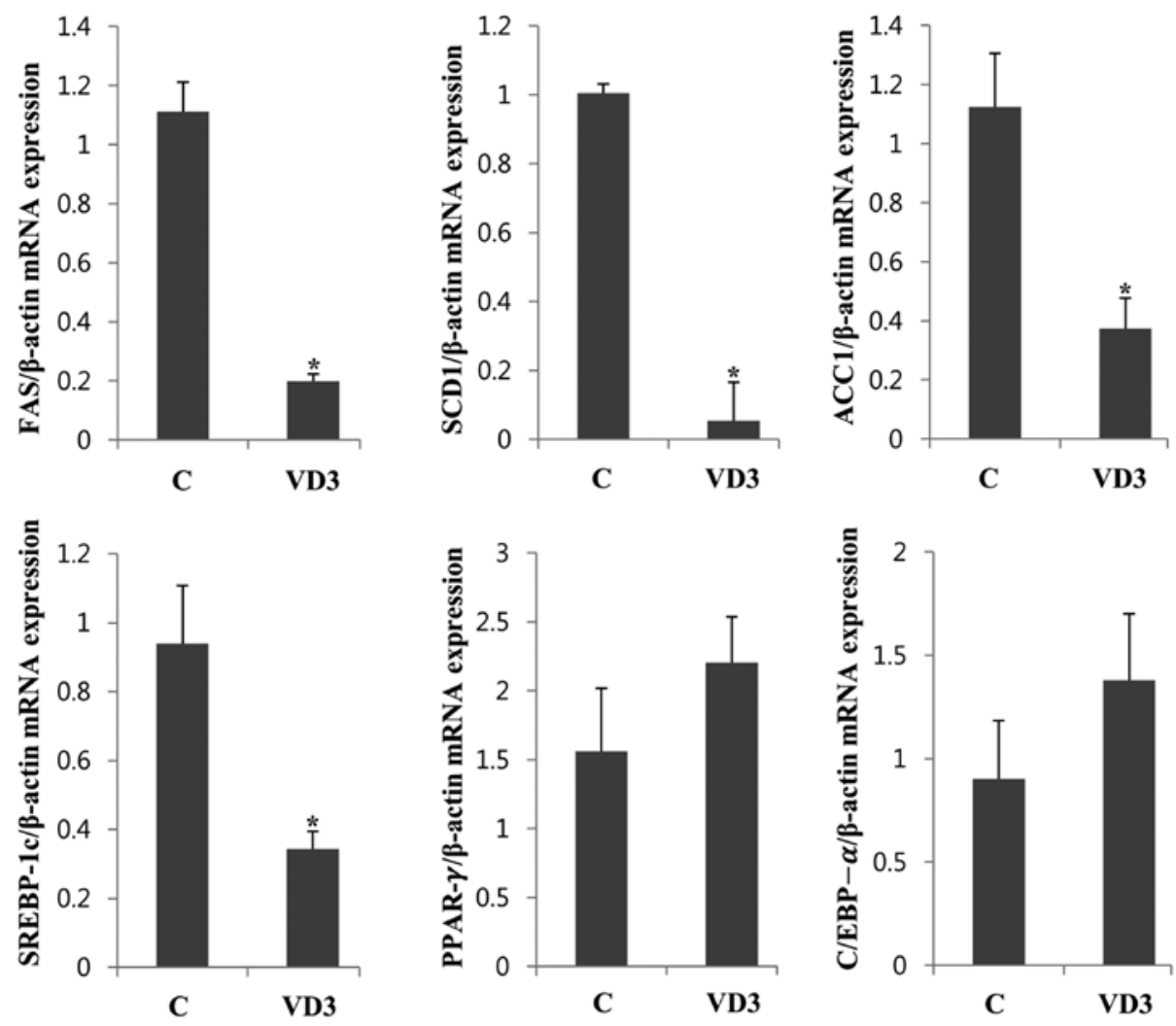

Figure 2. Effect of 1,25-dihydroxyvitamin D3 (VD3) on the mRNA levels of lipogenic genes in the liver of pregnant rats. Following treatment of the pregnant rats with VD3, mRNA was harvested from the liver. The transcriptional levels of fatty acid synthase (FAS), stearoyl-CoA desaturase 1 (SCD1), acetyl-CoA carboxylase 1 (ACC1), sterol regulatory element-binding protein-1c (SREBP-1c), peroxisome proliferator-activated receptor- $\gamma($ PPAR- $\gamma)$ and CCAAT/enhancerbinding protein- $\alpha(\mathrm{C} / \mathrm{EBP}-\alpha)$ were analyzed by RT-qPCR. Data are expressed as the means \pm SD from tissues of at least 3 rats. Gene expression was normalized to that of an internal control gene ( $\beta$-actin). ${ }^{*} \mathrm{P}<0.05$ compared to the control $(\mathrm{C}$; vehicle-treated) group.
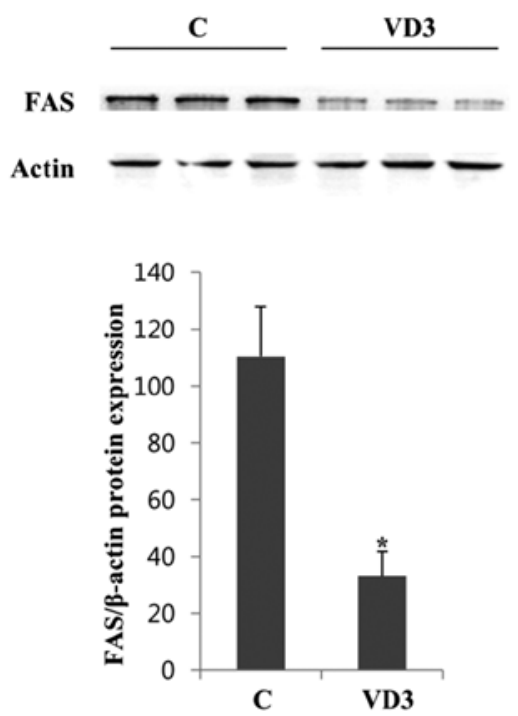
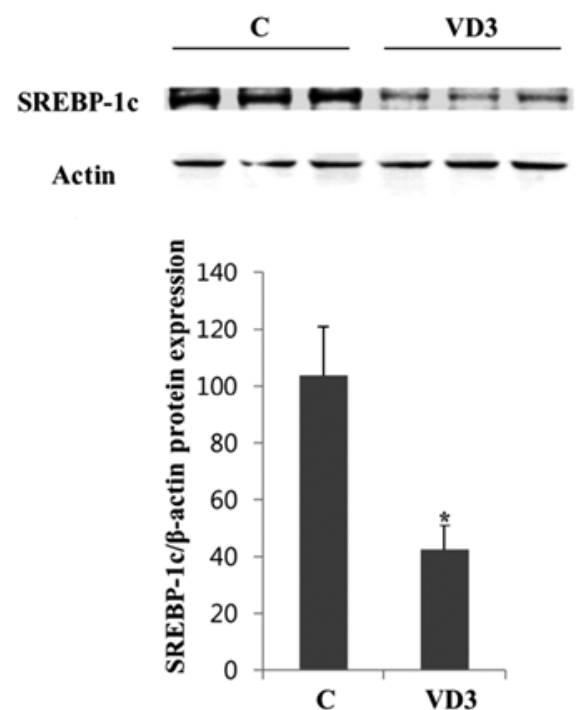
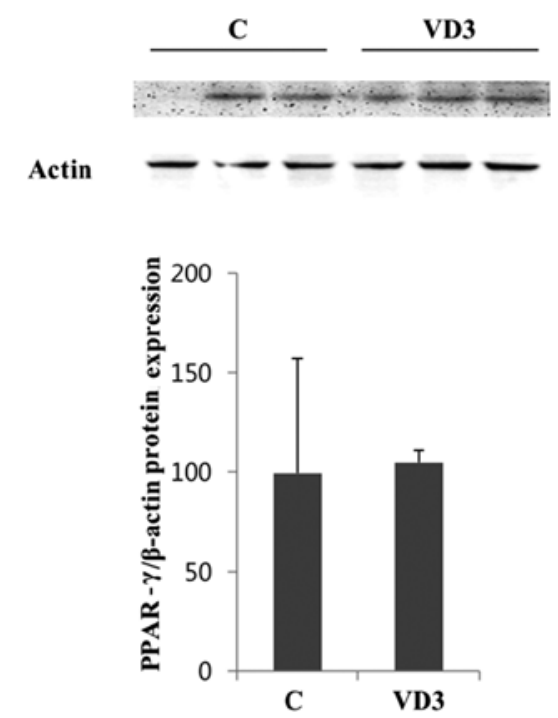

Figure 3. Effect of 1,25-dihydroxyvitamin D3 (VD3) on protein levels of lipogenic genes in the liver of pregnant rats. Following treatment of the pregnant rats with VD3, protein was harvested from the liver. The translational levels of fatty acid synthase (FAS), sterol regulatory element-binding protein-1c (SREBP-1c), and peroxisome proliferator-activated receptor- $\gamma(\operatorname{PPAR}-\gamma)$ were analyzed by western blot analysis. Data are expressed as the means \pm SD from tissues of at least 3 rats. Protein expression was normalized to that of an internal control protein ( $\beta$-actin). ${ }^{*} \mathrm{P}<0.05$ compared to the control (C; vehicle-treated) group.

lated the expression of INSIG2 in a tissue-specific manner. In the adipose tissue, VD3 markedly enhanced the INSIG2 mRNA levels, whereas in the liver, these levels were not significantly altered.

\section{Discussion}

The main natural source of vitamin $\mathrm{D}$ is synthesis from cholesterol in the skin; the dermal synthesis of vitamin D from 

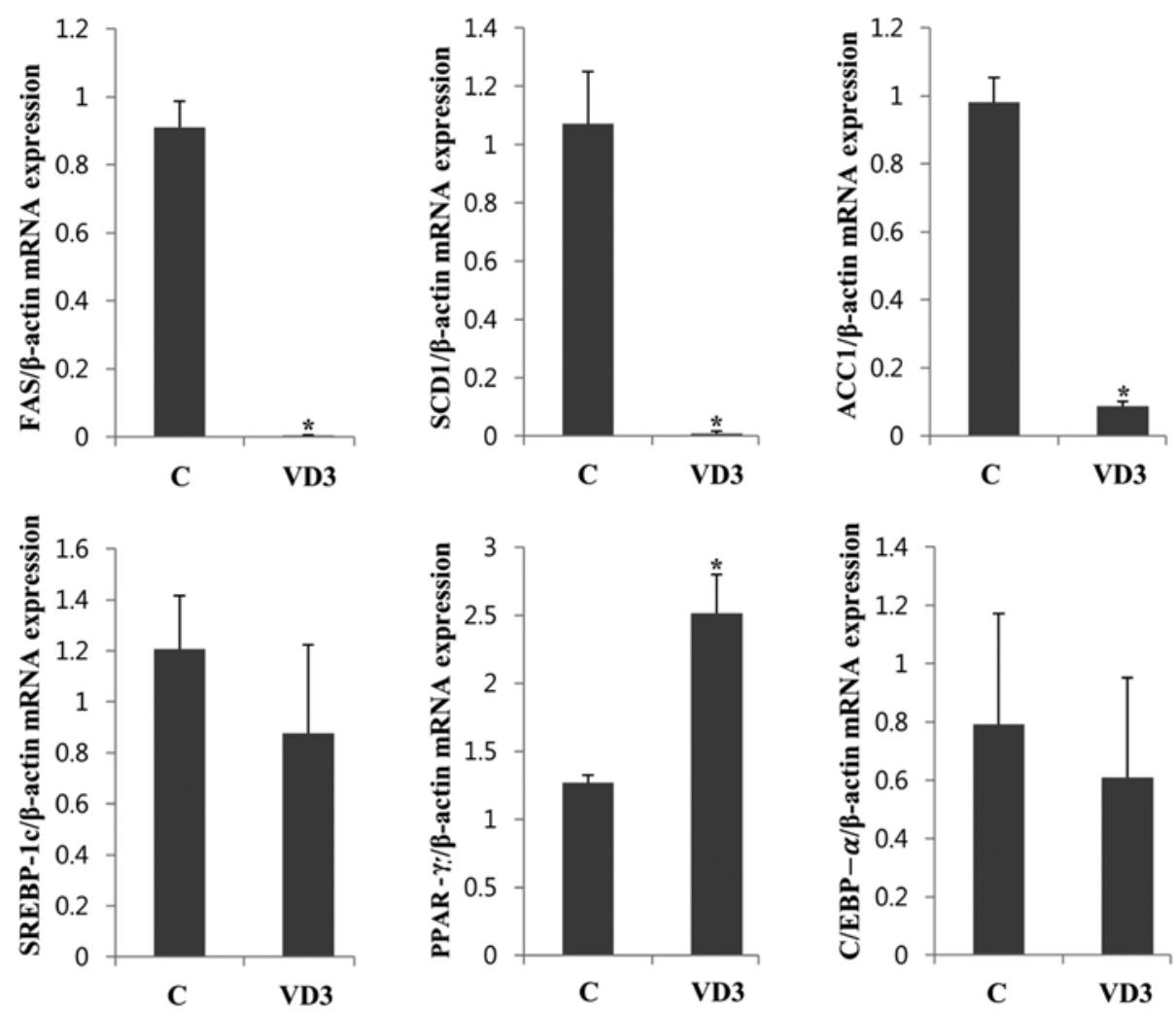

Figure 4. Effect of 1,25-dihydroxyvitamin D3 (VD3) on the mRNA levels of lipogenic genes in the adipose tissue of pregnant rats. Following treatment of the pregnant rats with VD3, mRNA was harvested from the adipose tissue. Transcriptional levels of fatty acid synthase (FAS), stearoyl-CoA desaturase 1 (SCD1), acetyl-CoA carboxylase 1 (ACC1), sterol regulatory element-binding protein-1c (SREBP-1c), peroxisome proliferator-activated receptor- $\gamma($ PPAR- $\gamma$ ) and CCAAT/enhancer-binding protein- $\alpha$ (C/EBP- $\alpha$ ) were analyzed by RT-qPCR. Data are expressed as the means \pm SD from tissues of at least 3 rats. Gene expression was normalized to that of an internal control gene ( $\beta$-actin). ${ }^{*} \mathrm{P}<0.05$ compared to the control (C; vehicle-treated) group.
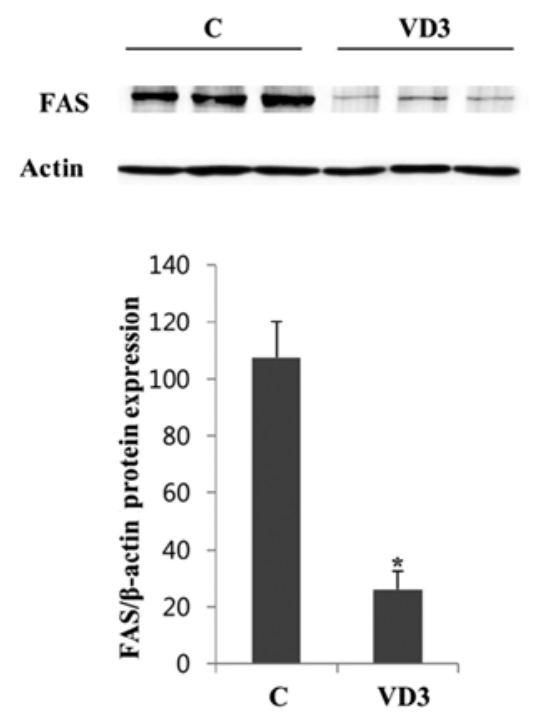
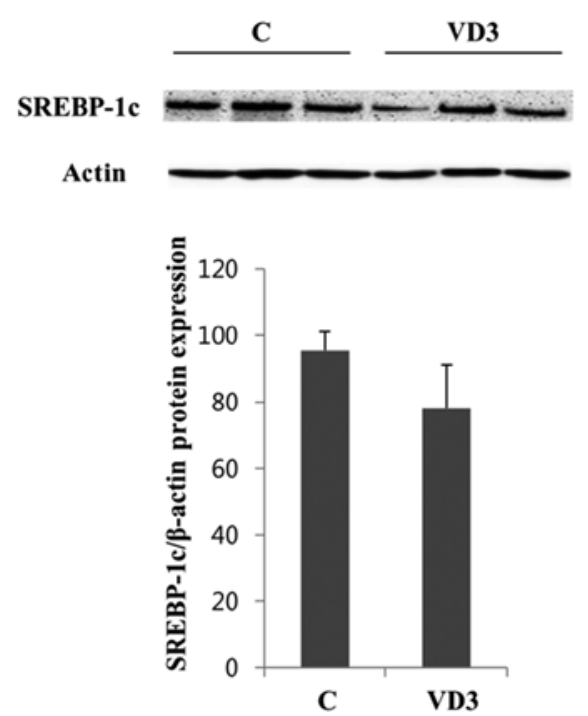
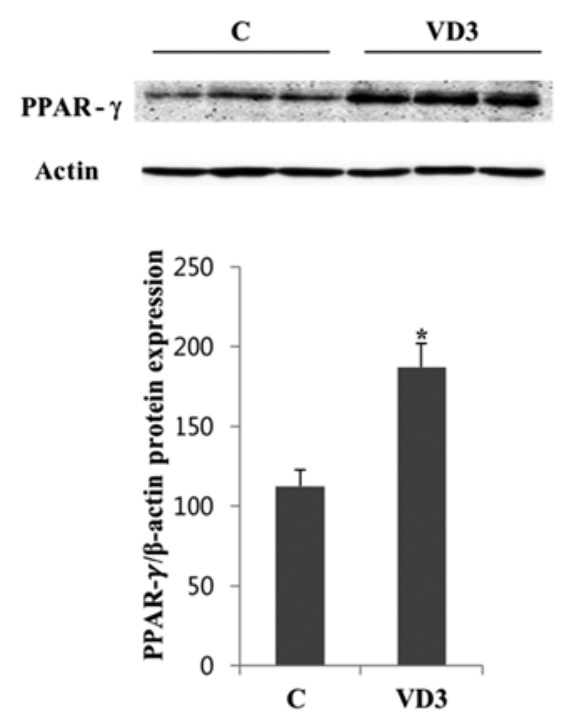

Figure 5. Effect of 1,25-dihydroxyvitamin D3 (VD3) on protein levels of lipogenic genes in the adipose tissue of pregnant rats. Following treatment of the pregnant rats with VD3, protein was harvested from adipose tissue. The translational levels of fatty acid synthase (FAS), sterol regulatory element-binding protein-1c (SREBP-1c), and peroxisome proliferator-activated receptor- $\gamma(\operatorname{PPAR}-\gamma)$ were analyzed by western blot analysis. Data are expressed as the means \pm SD from tissue of at least 3 rats. Protein expression was normalized to that of an internal control protein ( $\beta$-actin). ${ }^{*} \mathrm{P}<0.05$ compared to the control (C; vehicle-treated) group.

cholesterol is dependent on exposure to sunlight. Full-body exposure during the summer months for 10-15 min will provide 10,000 to $20,000 \mathrm{IU}$ of VD3 within $24 \mathrm{~h}$, for an adult (10).
However, the amount of UV exposure required for the synthesis of VD3 also depends on factors other than just the amount of time spent in the sun (29). Vitamin D deficiency is defined as a 

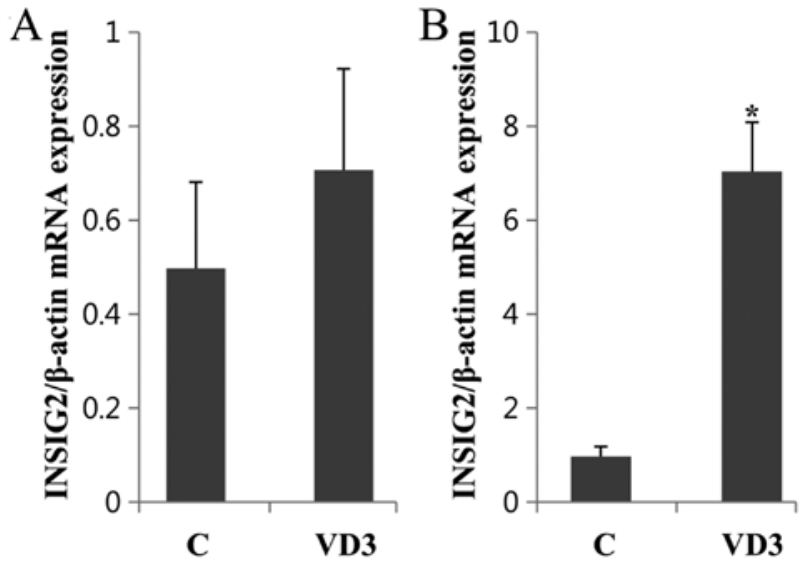

Figure 6. Tissue-specific effect of 1,25-dihydroxyvitamin D3 (VD3) on the mRNA levels of insulin-induced gene 2 (INSIG2) in the liver and adipose tissue. Following treatment of the pregnant rats with VD3, the translational levels of INSIG2 were analyzed by RT-qPCR. (A) Liver; (B) adipose tissue. Data are expressed as the means \pm SD from tissue of at least 3 rats. Gene expression was normalized to that of an internal control gene ( $\beta$-actin). ${ }^{*} \mathrm{P}<0.05$ compared to the control $(\mathrm{C}$; vehicle-treated) group.

concentration of $25(\mathrm{OH}) \mathrm{D}<30 \mathrm{nmol} / \mathrm{l}$. The Indoor Air Quality Act of 1989 reported that Americans spent $93 \%$ of their time indoors on average, thus contributing to a higher prevalence of lower $25(\mathrm{OH}) \mathrm{D}$ concentrations in the blood of adults. Vitamin D deficiency among African American children has also been reported (30).

It is known that VD3 inhibits lipogenesis in 3T3-L1 preadipocytes $(31,32)$. However, the majority of previous studies were performed in vitro using mouse or human pre-adipocytes, and only a few animal studies were in vivo. Yin et al demonstrated that VD3 modulates lipid metabolism by attenuating hepatic steatosis in the livers of male adult rats (33). The protective effect of VD3 against hepatic steatosis was mediated by downregulating SREBP-1c and its target genes, ACC and FAS (33). However, they did not examine the effect of VD3 in adipose tissue. In the present study, we, for the first time and to the best of our knowledge, examined the effects and role of VD3 in the adipose tissue and liver of pregnant animals. VD3 significantly reduced the fat deposition rate in adipose tissue and subsequently reduced the body weight of rats. As fat deposition and the size of adipocytes were reduced, we propose that VD3 administration alters lipogenesis.

In the present study, we found that the expression levels of the lipogenic proteins, FAS, SCD1 and ACC1, were downregulated by VD3 in both the liver and adipose tissue of pregnant rats (Fig. 3 and 5). These results suggest that the reduction of body weight and fat deposition following treatment with VD3 may be attributed to modulation of genes associated with lipogenesis. These lipogenic proteins are known to be regulated by specific transcription factors. For instance, the estrogen-mediated reduction of $\mathrm{ACC} 1$ and FAS gene expression is caused by the downregulation of SREBP-1c, which is a transcription factor that binds to the specific promoter sequence sterol regulatory element-1 (SRE1) (34). In addition, SREBP-1c regulates genes required for glucose metabolism, as well as fatty acid and lipid synthesis (35). PPAR- $\gamma$ and C/EBP- $\alpha$ are also transcription factors related to lipogenesis (36).
As SREBP-1c, PPAR- $\gamma$ and C/EBP- $\alpha$ have been reported to regulate the FAS, SCD1 and ACC1 genes $(37,38)$, we examined the expression of these transcription factors following treatment with VD3. The lipogenic transcription factors were not as strongly regulated by VD3 as the lipogenic enzymes. SREBP-1c was significantly downregulated by VD3 in the liver, but not significantly in the adipose tissue, whereas C/EBP- $\alpha$ expression was not significantly altered by VD3 in the liver or adipose tissue. Of note, PPAR $-\gamma$ was significantly upregulated by VD3 in adipose tissue, while its expression was not significantly increased in the liver. These results differ from those of previous studies on pre-adipocytes, which showed that VD3 markedly regulated SREBP-1c, C/EBP- $\alpha$ and PPAR- $\gamma$ expression $(13,31,39)$. It is possible that the mechanism of the VD3-mediated inhibition of lipogenesis is controlled in a different manner during pregnancy, as a result of the unique endocrine environment. According to a previous study, PPAR- $\gamma$ suppresses TG synthesis in rat hepatoma cells by reducing SREBP-1 activity through the upregulation of INSIG2 (40). INSIG2 is an endoplasmic reticulum protein that blocks the activation of SREBPs by binding to SREBP cleavage-activating protein (SCAP). Thus, in this study, we examined the expression levels of INSIG2 in the liver and adipose tissue following treatment with VD3. The INSIG2 mRNA levels were significantly increased following treatment with VD3 only in the adipose tissue. Taken together, our results suggest that VD3 reduces TG synthesis through the suppression of the expression of lipogenic enzymes in the liver and adipose tissue. However, the expression of the lipogenic transcription factors, SREBP-1c and PPAR- $\gamma$, was regulated by VD3 in a tissue-specific manner, and INSIG2 may be involved in this process.

Maternal obesity adversely affects the outcome of pregnancy, mainly as it increases the risk of hypertensive diseases (chronic hypertension and pre-eclampsia), gestational diabetes, the need for cesarean sections and the risk of infection (41). Furthermore, maternal obesity is related to a subsequent increased risk of childhood obesity and associated morbidity (41). Previous research has demonstrated the beneficial effects of exercise during pregnancy, including a reduced risk of gestational diabetes, pre-eclampsia, and a higher rate of operative vaginal deliveries (42). In addition, the number of obese women of reproductive age undergoing bariatric surgery is increasing. It has been reported that pregnancies following bariatric surgery are less likely to be complicated by gestational diabetes mellitus, hypertension, pre-eclampsia and macrosomia compared to obese women who have not undergone this surgery (43). However, physical activity during pregnancy is limited, and bariatric surgery is related to complications such as gastrointestinal obstruction and hemorrhage. Therefore, safe and easy methods to reduce maternal obesity during pregnancy are urgently required.

Taken together, the results of the present study demonstrated that VD3 downregulated lipogenesis-associated gene expression, which triggered a reduction in fat synthesis in the liver, suppressed the deposition of adipose tissue, and finally reduced the body weight of pregnant rats. These results suggest that the supplementation of VD3 or exposure to sunlight helps to control weight gain during pregnancy, and thereby prevents obesity-associated pregnancy complications. 


\section{Acknowledgements}

This study was supported by the 2012 Specialization Project Research Grant, funded by the Pusan National University and Pusan National University Research Grant, 2013.

\section{References}

1. Holick MF: McCollum Award Lecture, 1994: Vitamin D - new horizons for the 21st century. Am J Clin Nutr 60: 619-630, 1994.

2. Loomis WF: Skin-pigment regulation of vitamin-D biosynthesis in man. Science 157: 501-506, 1967.

3. Bouillon R, Okamura WH and Norman AW: Structure-function relationships in the vitamin D endocrine system. Endocr Rev 16: 200-257, 1995.

4. Dusso AS, Thadhani R and Slatopolsky E: Vitamin D receptor and analogs. Semin Nephrol 24: 10-16, 2004.

5. Carlberg C, Bendik I, Wyss A, Meier E, Sturzenbecker LJ, Grippo JF and Hunziker W: Two nuclear signalling pathways for vitamin D. Nature 361: 657-660, 1993.

6. Pike JW, Meyer MB and Martowicz ML: New techniques in transcription research extend our understanding of the molecular actions of the vitamin D hormone. IBMS BoneKEy 6: 169-180, 2009.

7. White JH: Vitamin D signaling, infectious diseases, and regulation of innate immunity. Infect Immun 76: 3837-3843, 2008.

8. Gorham ED, Garland CF, Garland FC, Grant WB, Mohr SB, Lipkin M, Newmark HL, Giovannucci E, Wei M and Holick MF: Vitamin D and prevention of colorectal cancer. J Steroid Biochem Mol Biol 97: 179-194, 2005.

9. Giovannucci E, Liu Y, Rimm EB, Hollis BW, Fuchs CS, Stampfer MJ and Willett WC: Prospective study of predictors of vitamin D status and cancer incidence and mortality in men. J Natl Cancer Inst 98: 451-459, 2006.

10. Holick MF: Vitamin D deficiency. N Engl J Med 357: 266-281, 2007.

11. Wortsman J, Matsuoka LY, Chen TC, Lu Z and Holick MF: Decreased bioavailability of vitamin D in obesity. Am J Clin Nutr 72: 690-693, 2000.

12. Parikh SJ, Edelman M, Uwaifo GI, Freedman RJ, SemegaJanneh M, Reynolds J and Yanovski JA: The relationship between obesity and serum 1,25-dihydroxy vitamin D concentrations in healthy adults. J Clin Endocrinol Metab 89: 1196-1199, 2004

13. Natarajan R: Vitamin D metabolites inhibit adipocyte differentiation in 3T3-L1 preadipocytes. Master's thesis, University of Massachusetts, 164, 2008.

14. Macotela Y, Boucher J, Tran TT and Kahn CR: Sex and depot differences in adipocyte insulin sensitivity and glucose metabolism. Diabetes 58: 803-812, 2009.

15. Kuhl J, Hilding A, Östenson CG, Grill V, Efendic S and Båvenholm P: Characterisation of subjects with early abnormalities of glucose tolerance in the Stockholm Diabetes Prevention Programme: the impact of sex and type 2 diabetes heredity. Diabetologia 48: 35-40, 2005

16. Catalano PM, Roman-Drago NM, Amini SB and Sims EA: Longitudinal changes in body composition and energy balance in lean women with normal and abnormal glucose tolerance during pregnancy. Am J Obstet Gynecol 179: 156-165, 1998.

17. Rasmussen KM and Yaktine AL: Weight gain during pregnancy: Reexamining the guidelines. National Academies Press, 2009.

18. Gillman MW, Rifas-Shiman S, Berkey CS, Field AE and Colditz GA: Maternal gestational diabetes, birth weight, and adolescent obesity. Pediatrics 111: e221-e226, 2003.

19. Parsons TJ, Power C, Logan S and Summerbell CD: Childhood predictors of adult obesity: a systematic review. Int J Obes Relat Metab Disord 23 (Suppl 8): S1-S107, 1999.

20. Rogers I; EURO-BLCS Study Group: The influence of birthweight and intrauterine environment on adiposity and fat distribution in later life. Int J Obes Relat Metab Disord 27: 755-777, 2003.

21. Chu SY, Callaghan WM, Kim SY, Schmid CH, Lau J, England LJ and Dietz PM: Maternal obesity and risk of gestational diabetes mellitus. Diabetes Care 30: 2070-2076, 2007.

22. O'Brien TE, Ray JG and Chan WS: Maternal body mass index and the risk of preeclampsia: a systematic overview. Epidemiology 14: $368-374,2003$
23. Chu SY, Kim SY, Schmid CH, Dietz PM, Callaghan WM, Lau J and Curtis KM: Maternal obesity and risk of cesarean delivery: a meta-analysis. Obes Rev 8: 385-394, 2007.

24. Haddow JE, Neveux LM, Palomaki GE, Lambert-Messerlian G, Canick JA, Grenache DG and Lu J: The relationship between PTH and 25-hydroxy vitamin D early in pregnancy. Clin Endocrinol (Oxf) 75: 309-314, 2011

25. Ginde AA, Sullivan AF, Mansbach JM and Camargo CA Jr: Vitamin D insufficiency in pregnant and nonpregnant women of childbearing age in the United States. Am J Obstet Gynecol 202: 436.e1-8, 2010.

26. Haagsman H, De Haas C, Geelen M and Van Golde L: Regulation of triacylglycerol synthesis in the liver. Modulation of diacylglycerol acyltransferase activity in vitro. J Biol Chem 257: 10593-10598, 1982

27. Lenhard JM: Lipogenic enzymes as therapeutic targets for obesity and diabetes. Curr Pharm Des 17: 325-331, 2011.

28. Farmer SR: Transcriptional control of adipocyte formation. Cell Metab 4: 263-273, 2006.

29. Wagner CL and Greer FR; American Academy of Pediatrics Section on Breastfeeding; American Academy of Pediatrics Committee on Nutrition: Prevention of rickets and vitamin D deficiency in infants, children, and adolescents. Pediatrics 122: 1142-1152, 2008

30. Nesby-O'Dell S, Scanlon KS, Cogswell ME, Gillespie C, Hollis BW, Looker AC, Allen C, Doughertly C, Gunter EW and Bowman BA: Hypovitaminosis D prevalence and determinants among African American and white women of reproductive age: third National Health and Nutrition Examination Survey, 1988-1994. Am J Clin Nutr 76: 187-192, 2002.

31. Blumberg JM, Tzameli I, Astapova I, Lam FS, Flier JS and Hollenberg AN: Complex role of the vitamin D receptor and its ligand in adipogenesis in 3T3-L1 cells. J Biol Chem 281: 11205-11213, 2006.

32. Lee S, Lee D-K, Choi E and Lee JW: Identification of a functional vitamin $\mathrm{D}$ response element in the murine Insig-2 promoter and its potential role in the differentiation of 3T3-L1 preadipocytes. Mol Endocrinol 19: 399-408, 2005.

33. Yin Y, Yu Z, Xia M, Luo X, Lu X and Ling W: Vitamin D attenuates high fat diet-induced hepatic steatosis in rats by modulating lipid metabolism. Eur J Clin Invest 42: 1189-1196, 2012.

34. D'Eon TM, Souza SC, Aronovitz M, Obin MS, Fried SK and Greenberg AS: Estrogen regulation of adiposity and fuel partitioning. Evidence of genomic and non-genomic regulation of lipogenic and oxidative pathways. J Biol Chem 280: 35983-35991, 2005.

35. Ferré $\mathrm{P}$ and Foufelle F: Hepatic steatosis: a role for de novo lipogenesis and the transcription factor SREBP-1c. Diabetes Obes Metab 12 (Suppl 2): 83-92, 2010.

36. Brun RP, Kim JB, Hu E and Spiegelman BM: Peroxisome proliferator-activated receptor gamma and the control of adipogenesis. Curr Opin Lipidol 8: 212-218, 1997.

37. Horton JD, Shimomura I, Brown MS, Hammer RE, Goldstein JL and Shimano H: Activation of cholesterol synthesis in preference to fatty acid synthesis in liver and adipose tissue of transgenic mice overproducing sterol regulatory element-binding protein-2. J Clin Invest 101: 2331-2339, 1998.

38. Shimano H, Horton JD, Shimomura I, Hammer RE, Brown MS and Goldstein JL: Isoform 1c of sterol regulatory element binding protein is less active than isoform 1a in livers of transgenic mice and in cultured cells. J Clin Invest 99: 846-854, 1997.

39. Kong J and Li YC: Molecular mechanism of 1,25-dihydroxyvitamin D3 inhibition of adipogenesis in 3T3-L1 cells. Am J Physiol Endocrinol Metab 290: E916-E924, 2006.

40. König B, Koch A, Spielmann J, Hilgenfeld C, Hirche F, Stangl GI and Eder K: Activation of PPARalpha and PPARgamma reduces triacylglycerol synthesis in rat hepatoma cells by reduction of nuclear SREBP-1. Eur J Pharmacol 605: 23-30, 2009.

41. Castro LC and Avina RL: Maternal obesity and pregnancy outcomes. Curr Opin Obstet Gynecol 14: 601-606, 2002.

42. Sui Z and Dodd JM: Exercise in obese pregnant women: positive impacts and current perceptions. Int J Womens Health 5: 389-398, 2013.

43. Sharara HA, Rhaman LNA, Ummunnisa F, Aboubaker N, Abdullah MM and Shaikh N: Obese nulliparous women and the risk for maternal and fetal complications. Open J Obstet Gynecol 4: 239-242, 2014. 